\section{Vaccinating people who have had covid-19: why doesn't natural immunity count in the US?}

An error occurred in the table of this article by Jennifer Block (BMJ 2021;374:n2101, doi:). The first row of the table includes children aged 0-17 not 5-17. The online version has been corrected. 